\title{
Sorafenib combined with radiation therapy for advanced hepatocellular carcinoma with portal and hepatic vein invasion extending to the inferior vena cava: a complete response case according to modified RECIST criteria
}

\author{
Yuri Cho', Bo Hyun Kim 1 , Tae Hyun Kim ${ }^{1,3}$, Young Hwan Koh ${ }^{4}$, Joong-Won Park ${ }^{1,2}$ \\ ${ }^{1}$ Center for Liver and Pancreatobiliary Cancer, ${ }^{2}$ Graduate School of Cancer Science and Policy, ${ }^{3}$ Center for Proton Therapy, \\ ${ }^{4}$ Department of Radiology, National Cancer Center, Goyang, Korea
}

Received Dec. 15, 2021

Revised Jan. 13, 2022

Accepted Jan. 18, 2022
The prognosis of patients with advanced hepatocellular carcinoma (HCC) with tumor thrombus extending to the inferior vena cava (IVC) is extremely poor. Herein, we present a rare case of advanced HCC that was treated with sorafenib and radiotherapy, leading to complete remission. This patient had a $9 \mathrm{~cm}$ infiltrative $\mathrm{HCC}$ occupying almost the entire left lobe with a tumor thrombus extending through the hepatic vein, IVC, and left portal vein. The patient received $400 \mathrm{mg}$ sorafenib twice daily. One year after the start of sorafenib, intensity-modulated radiation therapy for viable HCC and tumor thrombus was performed with a dose of 5,500 cGy. Twenty-seven months after the starting date of sorafenib, there was no intratumoral arterial enhancement, which suggested a complete response according to the modified RECIST criteria. This case suggests that the combination of sorafenib and radiotherapy might provide clinical benefits in patients with advanced HCC with IVC tumor thrombus. (J Liver Cancer 2022;22:63-68)

Keywords: Hepatocellular carcinoma; Sorafenib; Radiotherapy; Complete remission; Case report

\section{INTRODUCTION}

Hepatocellular carcinoma (HCC) often metastasizes to the lymph nodes, bones, and lungs. The prognosis is particularly poor for patients with HCC with tumor thrombi in the main vasculature, such as the portal vein, hepatic vein, and even inferior vena cava (IVC); the median overall survival (OS) of patients with IVC invasion is only 2-3 months, if untreated. ${ }^{1}$ Indeed, patients with HCC extending to the IVC may develop secondary Budd-Chiari syndrome, pulmonary infarction,

\section{Corresponding author: Joong-Won Park}

Center for Liver and Pancreatobiliary Cancer, National Cancer Center, 323 Ilsan-ro, Ilsandong-gu, Goyang 10408, Korea Tel. +82-31-920-1605, Fax. +82-31-920-2799 E-mail: jwpark@ncc.re.kr and lung metastasis. ${ }^{2}$

Systemic therapy is the standard treatment for HCC with vascular invasion. ${ }^{3}$ Sorafenib is an oral multikinase inhibitor which blocks the key mediators of hepatocarcinogenesis including Raf- 1 and vascular endothelial growth factor. ${ }^{4,5}$ Sorafenib is the first oral systemic agent for advanced or unresectable HCC.

Radiotherapy (RT) is one of the possible treatment options for patients with major vascular invasion. RT focused on macroscopic vascular invasion can reduce the extent of vascular invasion, leading to the restoration of blood flow to the non-tumor liver tissue. ${ }^{6}$ Some retrospective studies have suggested that RT is a feasible and safe option to palliate HCC with IVC invasion, with a 1 year OS rate of $53.6 \%$, a response rate of $59.2 \%$, and possible severe complication rate of $1.2 \%{ }^{7}$ 
RT may help prolong the OS of HCC patients with macrovascular invasion.

Here, we report an extremely rare case of initially unresectable advanced HCC with tumor thrombus extending to the IVC, treated with a combination of sorafenib and RT, resulting in a complete response (CR) after multidisciplinary treatment. This case report was described according to the CARE guidelines available from https://www.care-statement.org/.

\section{CASE REPORT}

A 48 year-old man presented with a large liver mass detected on ultrasonography screening at another hospital with epigastric pain. He had chronic hepatitis B; however, he was not under regular surveillance. Liver function was preserved with a Child-Pugh score of 5, and the Eastern Cooperative Oncology Group performance status was 1. Initial liver dynamic computed tomography (CT) revealed an approximately $9 \mathrm{~cm}$ infiltrative HCC occupying almost the entire left lobe with a tumor thrombus extending through the hepatic vein, the IVC and the left portal vein (Fig. 1).

The patient was diagnosed with advanced-stage HCC (Barcelona Clinic Liver Cancer C; modified Union for International Cancer Control T4N0M0, stage IVa) without liver biopsy based on typical imaging findings in December 2010. Blood tests showed a white blood cell count of $4,870 / \mathrm{mm}^{3}$, a hemoglobin level of $14.2 \mathrm{~g} / \mathrm{dL}$, platelet count of $123,000 / \mu \mathrm{L}$, aspartate aminotransferase concentration of $72 \mathrm{IU} / \mathrm{L}$, alanine aminotransferase concentration of $64 \mathrm{IU} / \mathrm{L}$, the total protein level of $6.7 \mathrm{~g} / \mathrm{dL}$, total albumin level of $3.6 \mathrm{~g} / \mathrm{dL}$, prothrom-
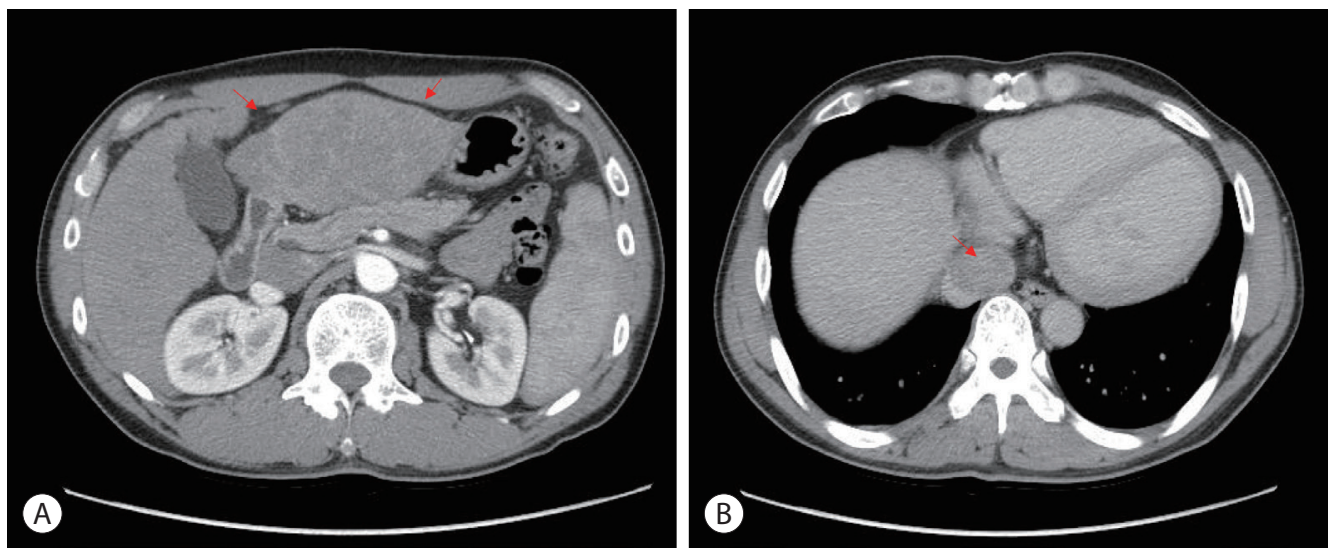

Figure 1. Initial liver dynamic computed tomography imaging findings. (A) Infiltrative hepatocellular carcinoma, left lobe lateral section with left hepatic vein in the arterial phase (red arrows). (B) Inferior vena cava tumor thrombus in the delayed phase (red arrow).
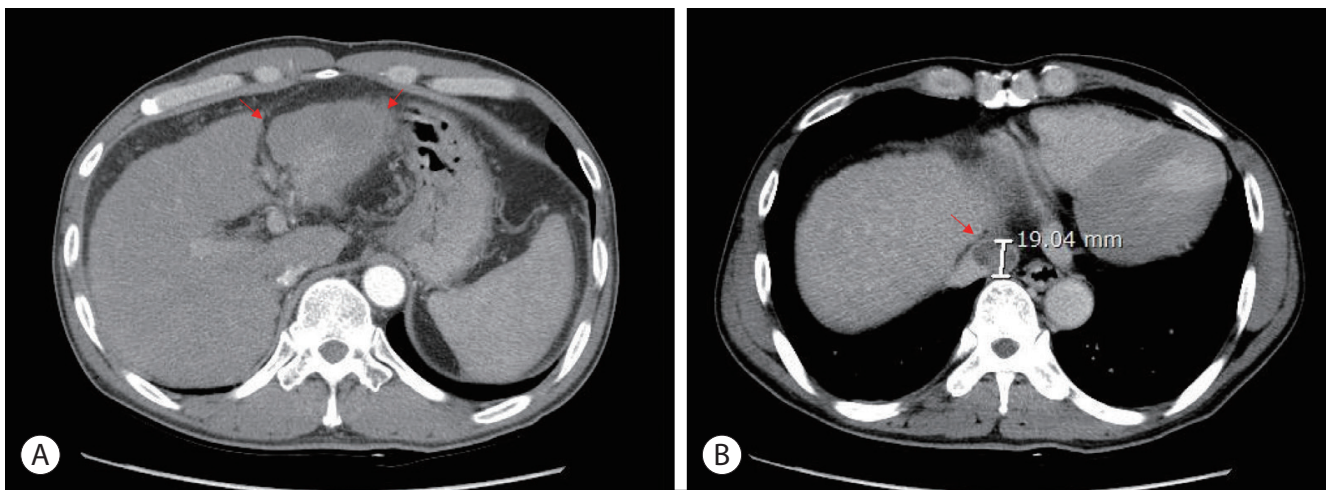

Figure 2. Follow-up liver dynamic computed tomography imaging findings after 1 year of sorafenib treatment. (A) Decreased size of infiltrative hepatocellular carcinoma, left lobe lateral section with left hepatic vein in the arterial phase (red arrows). (B) Decreased size and mass effect of inferior vena cava tumor thrombus in the delayed phase (red arrow). 
bin time of $92 \%$, and total bilirubin concentration of 0.8 $\mathrm{mg} / \mathrm{dL}$. His serum hepatitis B virus DNA level was 545,671 $\mathrm{IU} / \mathrm{mL}$ with hepatitis B e-antigen positivity. The patient was treated with $0.5 \mathrm{mg}$ of entecavir per day. The initial level of alpha-fetoprotein (AFP) was $10.2 \mathrm{ng} / \mathrm{mL}$, and the protein induced by the absence of vitamin K or antagonist-II (PIVKAII) was $>2,000 \mathrm{mAU} / \mathrm{mL}$. First, we planned systemic treatment with sorafenib. The patient was treated with sorafenib $400 \mathrm{mg}$ twice daily, beginning in December 2010. One year after the start of sorafenib, the main mass of the left lobe lateral section was reduced with decreasing arterial enhance- ment. The left portal vein tumor thrombus, which appeared to be in the umbilical portion, also decreased in extent compared with the initial CT. The mass effect of the tumor thrombus extending to the IVC was also reduced (Fig. 2). Then, intensity-modulated radiation therapy with simultaneous integrated boost for viable HCC and tumor thrombus was performed with a dose of 5,500 cGy with 22 fractions for planning target volume (PTV)-1 and 4,400 cGy with 22 fractions for PTV-2 (Fig. 3). Twenty-seven months after the starting date of sorafenib, there was no intratumoral arterial enhancement in all target lesions and no hot uptake in posi-
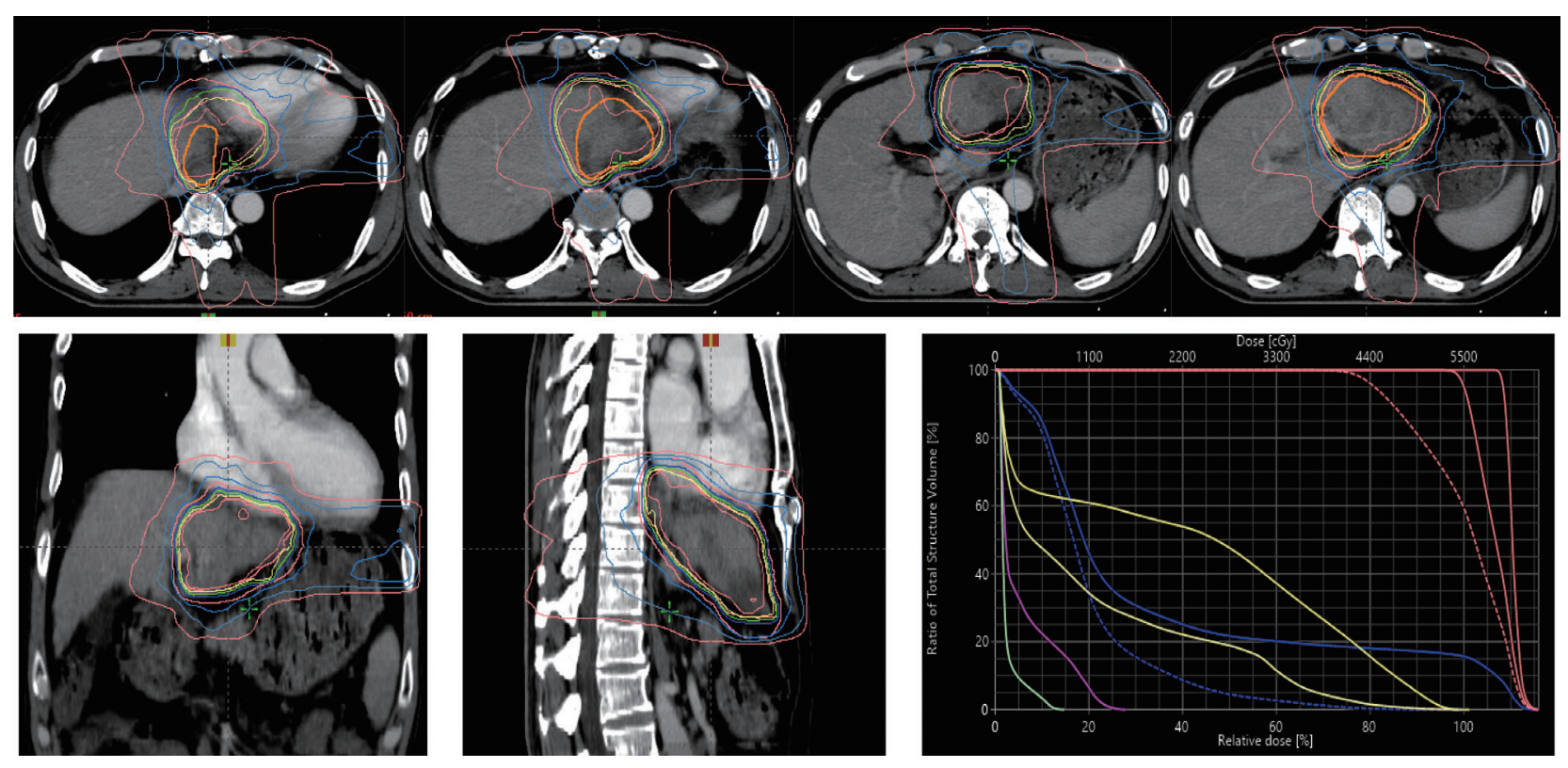

Figure 3. Intensity modulated radiation therapy with Simultaneous integrated boost radiotherapy. Prescription dose to target volumes: planning target volume (PTV)-1, 5,500 cGy with 22 fractions; PTV-2, 4,400 cGy with 22 fractions.
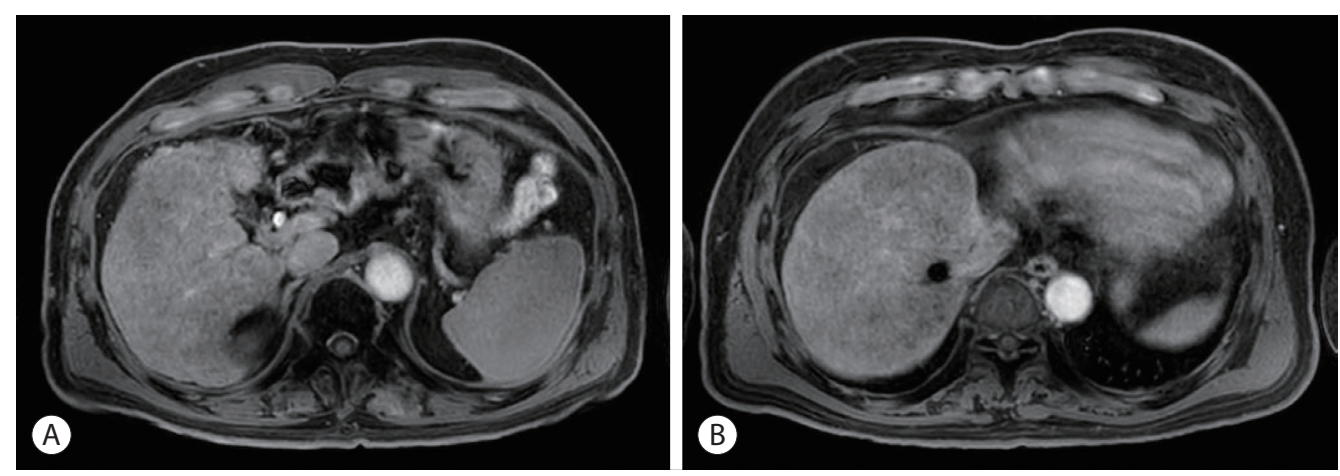

Figure 4. Follow-up liver magnetic resonance imaging on hepatobiliary phase on May 2021. (A) Obliteration of Lt. portal vein and left hepatic vein in the hepatobiliary phase. (B) No tumor arterial phase enhancement and radiation-induced parenchymal change in atrophic liver left lateral section in the hepatobiliary phase. 


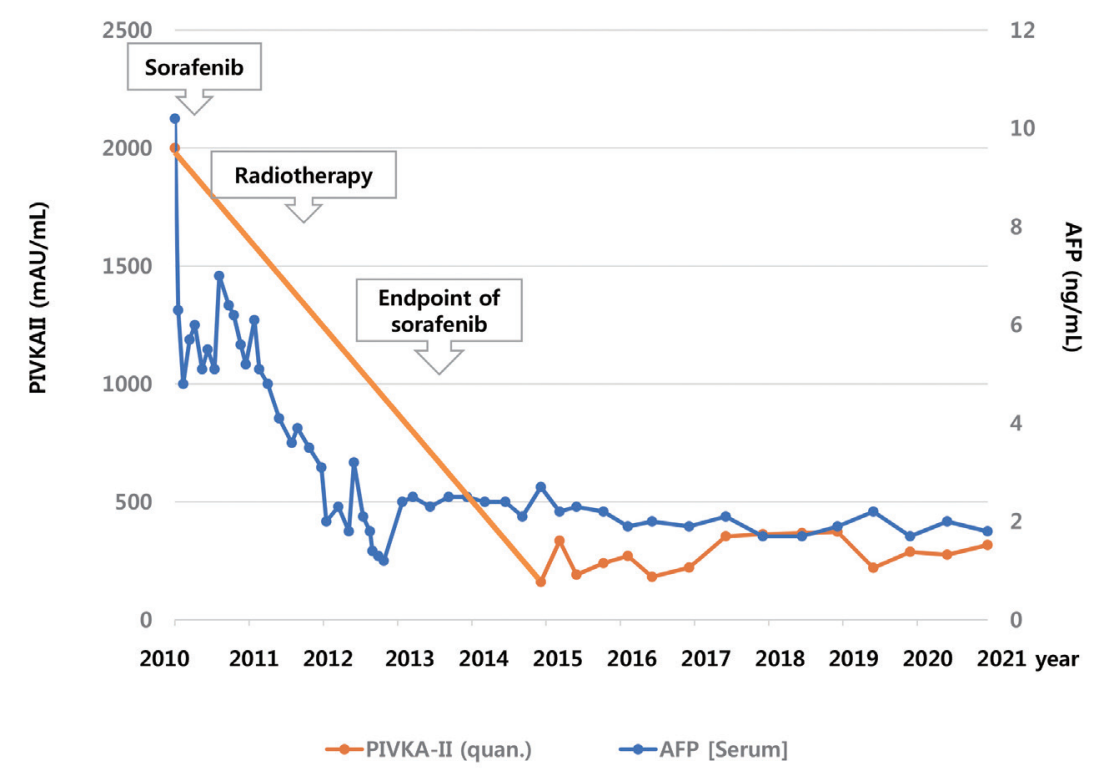

Figure 5. Clinical course of the patient with alpha-fetoprotein (AFP), prothrombin induced by vitamin K absence (PIVKA)-II.

tron emission tomography/CT on the left liver, which suggested CR according to the modified response evaluation criteria in solid tumors criteria version 1.1. In August 2013, the total bilirubin level increased to $5.1 \mathrm{mg} / \mathrm{dL}$. Subsequently, sorafenib was discontinued after 127 weeks of treatment. During the follow-up period, 10.6 years from initiation of sorafenib, there was still no intratumoral arterial enhancement. On the last follow-up liver magnetic resonance imaging in July 2021, there was no tumor arterial phase enhancement (Fig. 4). RT-induced parenchymal changes in the atrophic left lateral section were noted, and the left portal vein and left hepatic vein were obliterated with a contracted thrombus. The level of AFP was $2.0 \mathrm{ng} / \mathrm{mL}$, and the PIVKAII level was $276 \mathrm{mAU} / \mathrm{mL}$ in May 2021 (Fig. 5).

\section{DISCUSSION}

The presence of IVC tumor thrombus is one of the most significant factors for poor prognosis in HCC patients. In this case, the initial liver function was well preserved with antiviral treatment, and partial remission was achieved 1 year of sorafenib treatment. The patient received RT at that time, when the tumor burden was reduced and the tumor extent was relatively localized. Intensity-modulated radiotherapy using a simultaneous integrated boost technique also facilitated safe and effective delivery of high doses to tumors. After approximately three years of long-term sorafenib therapy, his liver function decreased, presenting with hyperbilirubinemia and increased ascites. Sorafenib was discontinued after 127 weeks of treatment. CR status has been maintained since then. This case suggests that the combination of sorafenib and RT might provide clinical benefits in patients with advanced HCC who have macrovascular invasion.

The rationale for combining radiotherapy with sorafenib is targeting the RAS-RAF-MAPK and VEGFR signaling pathways, which are specifically activated after exposure to radiation and responsible for radioresistance phenomena. Sorafenib and RT represent complementary strategies, as RT may be useful to prolong the effect of sorafenib through the control of macroscopic disease, when sorafenib may target latent microscopic disease.

Sorafenib may contribute to enhancing the radiosensitivity of HCC by improving oxygenation of tumor. Moreover, the antiproliferative effects of sorafenib may delay tumor progression outside the RT field. RT also may improve the overall response by reducing tumor burden. Therefore, combination of sorafenib and RT might be an effective and novel therapeutic strategy with improved antitumor effects. 
Cha et al. reported the feasibility of sorafenib combined with local RT in advanced HCC patients with macrovascular invasion or distant metastasis. They analyzed 31 patients who were treated with a daily dose of $800 \mathrm{mg}$ of sorafenib and radiotherapy, showing an in-field response rate of $100 \%$ in the primary group who received RT for primary liver lesions (13 patients) according to the modified RECIST. ${ }^{8}$ Their median OS was 7.8 months, and there were no cases of radiation-induced hepatic toxicity. However, three patients (17\%) experienced hand-foot syndrome with grades 3-4. There was one case of grade 3 duodenal bleeding that was manageable after conservative care.

Chen et al. ${ }^{9}$ reported a phase 2 study of combined sorafenib and RT in patients with advanced HCC. Among 40 patients, $22(55.0 \%)$ achieved CR or partial remission at the initial assessment, and 18 (45\%) had stable or progressive disease. The 2 year OS and in-field progression-free survival rates were $32 \%$ and $39 \%$, respectively. ${ }^{9}$ Six patients (15\%) developed treatment-related grade $\geq 3$ hepatic toxicity during the sequential phase, and three of them were fatal.

In an effort to improve outcomes in advanced HCC, the combination of systemic therapy and RT is an attractive strategy, which may result in better long-term control. The mechanisms of additive effects combining these modalities are complex and include decreased tumor hypoxia and radiosensitization of endothelial cells. The antiangiogenic effect of sorafenib might result in a relative normalization of blood flow in tumors with more efficient delivery of oxygen and therapeutic agents to the target HCC cells. ${ }^{10}$ Regarding the timing of radiotherapy, it is not clear whether concurrent or sequential radiotherapy leads to a better outcome. Many clinical trials have been performed to evaluate the efficacy and safety of a combination of systemic therapy and radiotherapy for HCC, ${ }^{9,11,12}$ however, the evidence for the best timing is insufficient. In this case, radiotherapy was decided at a point in time when the extent of the tumor was relatively localized, and the benefit of radiotherapy was judged to outweigh the risk.

Based on this case, the use of systemic therapy in combination with RT might prolong the OS of patients with IVC tumor thrombus. Further elucidation of the mechanisms of in- teraction between sorafenib and RT and the clinical implications for macrovascular invasion are also warranted.

\section{Conflict of Interest}

The authors have no conflicts of interest to disclose.

\section{Ethics Statement}

The Institutional Review Board of National Cancer Center waived the requirement for ethics approval and informed consent (IRB number: 2021-0729-0001).

\section{Funding Statement}

This research was supported by a grant of the Korea Health Technology R\&D Project through the Korea Health Industry Development Institute, funded by the Ministry of Health \& Welfare, Korea (HI21C0240), and by the National Research Foundation of Korea grant funded by the Korea government (2021R1A2C4001401).

\section{Data Availability}

Data sharing not applicable to this article as no datasets were generated or analyzed for this case report.

\section{ORCID}

$\begin{array}{ll}\text { Yuri Cho } & \text { https://orcid.org/0000-0002-4488-5352 } \\ \text { Bo Hyun Kim } & \text { https://orcid.org/0000-0001-8355-3126 } \\ \text { Tae Hyun Kim } & \text { https://orcid.org/0000-0001-8413-3385 } \\ \text { Young Hwan Koh } & \text { https://orcid.org/0000-0002-4598-6956 } \\ \text { Joong-Won Park } & \text { https://orcid.org/0000-0001-9972-0494 }\end{array}$

\section{Author Contribution}

Conceptualization: JWP

Data curation: YC, BHK, JWP

Methodology: THK, YHK, JWP

Project administration: YC, JWP

Writing original draft: YC, JWP

Writing review \& editing: YC, JWP

Approval of final manuscript: all authors 


\section{References}

1. Chun YH, Ahn SH, Park JY, Kim DY, Han KH, Chon CY, et al. Clinical characteristics and treatment outcomes of hepatocellular carcinoma with inferior vena cava/heart invasion. Anticancer Res 2011;31:4641-4646.

2. Okada S. How to manage hepatic vein tumour thrombus in hepatocellular carcinoma. J Gastroenterol Hepatol 2000;15:346-348.

3. Korean Liver Cancer Association; National Cancer Center. 2018 Korean Liver Cancer Association-National Cancer Center Korea practice guidelines for the management of hepatocellular carcinoma. Gut Liver 2019;13:227-299.

4. Wilhelm SM, Carter C, Tang L, Wilkie D, McNabola A, Rong H, et al. BAY 43-9006 exhibits broad spectrum oral antitumor activity and targets the RAF/MEK/ERK pathway and receptor tyrosine kinases involved in tumor progression and angiogenesis. Cancer Res 2004;64:7099-7109.

5. Chang YS, Adnane J, Trail PA, Levy J, Henderson A, Xue D, et al. Sorafenib (BAY 43-9006) inhibits tumor growth and vascularization and induces tumor apoptosis and hypoxia in RCC xenograft models. Cancer Chemother Pharmacol 2007;59:561-574.

6. Kim YJ, Jung J, Joo JH, Kim SY, Kim JH, Lim YS, et al. Combined transarterial chemoembolization and radiotherapy as a first-line treatment for hepatocellular carcinoma with macroscopic vascular invasion: necessity to subclassify Barcelona Clinic Liver Cancer stage C. Radiother Oncol 2019;141:95-100.

7. Rim CH, Kim CY, Yang DS, Yoon WS. External beam radiation therapy to hepatocellular carcinoma involving inferior vena cava and/ or right atrium: a meta-analysis and systemic review. Radiother Oncol 2018;129:123-129.

8. Cha J, Seong J, Lee IJ, Kim JW, Han KH. Feasibility of sorafenib combined with local radiotherapy in advanced hepatocellular carcinoma. Yonsei Med J 2013;54:1178-1185.

9. Chen SW, Lin LC, Kuo YC, Liang JA, Kuo CC, Chiou JF. Phase 2 study of combined sorafenib and radiation therapy in patients with advanced hepatocellular carcinoma. Int J Radiat Oncol Biol Phys 2014;88:1041-1047.

10. Frankfurt O, Rosen ST. Mechanisms of glucocorticoid-induced apoptosis in hematologic malignancies: updates. Curr Opin Oncol 2004;16:553-563.

11. Wild AT, Gandhi N, Chettiar ST, Aziz K, Gajula RP, Williams RD, et al. Concurrent versus sequential sorafenib therapy in combination with radiation for hepatocellular carcinoma. PLoS One 2013;8:e65726.

12. Zhao JD, Liu J, Ren ZG, Gu K, Zhou ZH, Li WT, et al. Maintenance of Sorafenib following combined therapy of three-dimensional conformal radiation therapy/intensity-modulated radiation therapy and transcatheter arterial chemoembolization in patients with locally advanced hepatocellular carcinoma: a phase I/II study. Radiat Oncol 2010;5:12. 